\title{
Bladder Cancer pMX TNM Finding v6
}

National Cancer Institute

\section{Source}

National Cancer Institute. Bladder Cancer pMX TNM Finding v6. NCI Thesaurus. Code C61224.

Bladder cancer in which distant metastasis cannot be assessed. (from AJCC 6th Ed.) 